\title{
Sasa quelpaertensis Nakai extract induces p53-independent apoptosis via the elevation of nitric oxide production in human HCT116 colon cancer cells
}

\author{
MIN YOUNG KIM \\ Toxicology Laboratory, Faculty of Biotechnology (Biomaterials), College of Applied Science, \\ Jeju National University, Jeju 63243, Republic of Korea \\ Received September 23, 2019; Accepted January 6, 2020
}

DOI: $10.3892 / \mathrm{ol} .2020 .11379$

\begin{abstract}
Induction of apoptosis in human cancer cells by Sasa quelpaertensis Nakai has been considered to be a potential therapeutic target for cancer treatment; however, the underlying mechanisms of action are not well understood. The present study investigated the role of nitric oxide (NO*) and inhibitors of apoptosis (IAPs) during apoptosis induced by Sasa quelpaertensis Nakai extracts (SQE) in p53-wild type (WT) and p53-null HCT116 colon carcinoma cells. Trypan blue exclusion and Annexin V/propidium iodide assays were used to test for antiproliferation, and apoptosis and cell cycle. Griess and reverse transcription-polymerase chain reaction and western blotting assays were carried out to assay $\mathrm{NO}^{*}$ production, and to detect the mRNA and protein levels of Bcl-2, PARP and IAPs. A colorimetric assay was utilized to measure the time-dependent increase in caspase- 3 activity. SQE inhibited cell growth and promoted apoptosis by the elevation of $\mathrm{NO}^{\circ}$ in a dose- and time-dependent manner. In addition, both cell types underwent a reduction in mRNA and protein levels of IAPs (survivin, CIAP-1 and -2, and X-linked inhibitor of apoptosis) as well as anti-apoptotic Bcl-2, whereas an increase in protein expression of poly (ADP-ribose) polymerase 1 and caspase 3 activity was observed; however, an equivalent cytotoxic and apoptotic effect by SQE was observed in p53-WT and p53-null cells. Collectively, the results indicated that SQE-induced apoptosis was independent of p53 status and associated with modulation of endogenous $\mathrm{NO}^{\circ}$ and IAP family gene expression.
\end{abstract}

Correspondence to: Professor Min Young Kim, Toxicology Laboratory, Faculty of Biotechnology (Biomaterials), College of Applied Science, Jeju National University, Ara-1 dong, Jeju 63243, Republic of Korea

E-mail: jeffmkim@jejunu.ac.kr

Key words: Sasa quelpaertensis Nakai, apoptosis, p53, nitric oxide, inhibitors of apoptosis, HCT116 cells

\section{Introduction}

Colon cancer is the third most common diagnosed cancer with 1.8 million new cases in 2018 throughout the world, with a poor prognosis (1). The pathogenesis of colorectal cancer is complicate and multifactorial, and therefore difficult to diagnose in the earlier stage (2). Although surgical resection remains the only curative treatment for colon cancer, an alternative approach to reduce the mortality rate is chemotherapy (3). For many years, 5-fluorouracil (5-FU) is the most common used chemotherapy drug for colon cancer (4). Like other chemotherapeutics, however, it affects not only the cancer cells but also normal cells. As the dose increase of 5-FU, the side effects of the drug increase and resistance to the drug develops frequently. Thus, new strategies for the use of various natural products of plant origin in chemotherapy to minimize toxicity and drug side-effects; over $60 \%$ of anticancer drugs in use today are of natural origin (5). In combination with chemotherapy agents, natural compounds have led to not only reduce risk of drug adverse effects but can also improve the effectiveness of medication (5). Natural compounds possess the ability to modulate signaling pathways and regulated cell cycle-regulated gene expression, cell differentiation and apoptosis (5). Moreover, a lot of natural compounds are well tolerated by humans. Although some natural compounds have anticancer properties (6), cellular and molecular mechanisms involved in their anticancer activity is still unclear.

Sasa quelpaertensis Nakai is a species of bamboo grass native to South Korea and only grown on Halla mountain of Jeju Island. Like other bamboo species, S. quelpaertensis Nakai has been used in herbal medicine like other bamboo species for various pharmacological properties such as antioxidant (7-9), anticancer $(10,11)$, antidiabetic (12), inhibition of tyrosinase and melanin production $(13)$, hepatoprotective $(9,14)$ and anti-inflammatory (15) properties. Recently, S. quelpaertensis Nakai has earn commercial attention for its ability to prohibit human leukemia HL-60 cells $(16,17)$, gastric adenocarcinoma MKN-74 (17), colon cancer HT-29 (18) cells proliferation by inducing apoptosis. In those study, an excessive and unregulated nitric oxide ( $\mathrm{NO}^{\circ}$ ) synthesis has been implicated to abrogation of tumorigenicity and induction of apoptosis in tumor cells $(18,19)$. These results might be possible due to enhancing effect of iNOS 
gene stimulated by an S. quelpaertensis Nakai which may lead to excess cellular levels of $\mathrm{NO}^{*}$ which might be responsible for damage of proteins, nucleic acids, membranes and organelles, which can lead to activation of cell death processes such as apoptosis $(20,21)$. Nevertheless, only few reports are available regarding anticancer and antiproliferative activity of S. quelpaertensis Nakai, the precise action of S. quelpaertensis Nakai on apoptotic mechanism is less well understood.

In the present study, we aimed to investigate the effects of $S$. quelpaertensis Nakai to modulate NO signaling in human colon cancer cells as a possible mechanism underlying S. quelpaertensis Nakai-induced apoptosis. Furthermore, expression analysis of different mRNA and proteins involved in regulation of S. quelpaertensis Nakai-induced apoptotic signaling pathway based on p53 status was also carried out by employing two p53 isogenic HCT116 cell lines, p53 wildtype (p53-WT) and p53-deficent (p53-null) cells.

\section{Materials and methods}

Cell cultures and chemicals. Two isogenic HCT116 human colon carcinomas, wild-type p53 (p53-WT) and complete knockout of p53 (p53-null) cells, kindly gifted by Prof. Gerald N. Wogan (Massachusetts Institute of Technology), were maintained at $37^{\circ} \mathrm{C}$ with 5\% carbon dioxide in McCoy's 5A medium (cat. no. 12-168F) supplemented with $10 \%$ fetal bovine serum (cat. no. 35-015-CV, Corning, NY, USA), 100 units/ml penicillin (cat. no. 17-602E), $100 \mu \mathrm{g} / \mathrm{ml}$ streptomycin (cat. no. 17-602E) and $2 \mathrm{mM} \mathrm{L-glutamine} \mathrm{(cat.} \mathrm{no.} \mathrm{17-605E).} \mathrm{Reagents} \mathrm{and} \mathrm{cell}$ culture materials were purchased from the following sources: Cell culture reagents, Lonza; annexin V-FITC apoptotic assay kit (cat. no. 630109) and ApoAlert caspase-3 colorimetric assay kit (cat. no. 630217), Clontech Laboratories; $\mathrm{ECL}^{\mathrm{TM}}$ western blotting detection reagents (cat. no. RPN2209), GE Healthcare Bio-Sciences; RIPA lysis buffer (cat. no. R2002), Biosesang; anti-bcl-2 antibody (cat. no. OP91), Calbiochem; anti-actin antibody (C4, cat. no. sc-47778) and the secondary goat anti-rabbit (cat. no. sc-2004) or anti-mouse (cat. no. sc-2005) IgG conjugated to horseradish peroxidase, Santa Cruz Biotechnology; anti-survivin antibody (cat. no. 2803), anti-CIAP-1 antibody (cat. no. 4952), anti-CIAP-2 antibody (cat. no. 3130) and anti-XIAP antibody (cat. no.R2042), Cell Signaling Technology.

Preparation of S. quelpaertensis Nakai and its extracts. The leaves of S. quelpaertensis Nakai used for the present study were plucked during February 2012 from Mt. Halla on Jeju island in South Korea, and a voucher sample preserved for reference in the herbarium of Jeju National University. Fresh leaves were washed with water, drained, dried and powdered. Dried S. quelpaertensis Nakai leaf $(25 \mathrm{~g})$ was extracted with $70 \%$ ethanol $(250 \mathrm{ml})$ on a rotary shaker for $24 \mathrm{~h}$ and filtered with a Sep-Pak $\mathrm{C}_{18}$ cartridge and a $0.45 \mu \mathrm{m}$ membrane filter (Waters Corporation). The extracted liquid was concentrated using a rotary vacuum evaporator (Buchi Rotavapor R-200; Sigma-Aldrich), freeze dried and kept at $-20^{\circ} \mathrm{C}$ until used for experiment. The extraction yield of ethanol extracts was determined to be $11.8 \pm 0.13 \%$.

Assessment of cell viability. HCT 116 cells were seeded at $1 \times 10^{6}$ cells/well into 6 -well flat-bottom tissue culture plates the day before treatment. Cells were cultured for 24, 48 and $72 \mathrm{~h}$ in McCoy's 5A medium containing 0, 25, 50,100 and $200 \mu \mathrm{g} / \mathrm{ml}$ of $S$. quelpaertensis Nakai extracts. HCT116 cell viability was determined $24 \mathrm{~h}$ after treatment was determined by trypan blue exclusion. Cell viability was calculated as relative to control cells frown in culture medium with DMSO.

Apoptosis analysis. After treatment with $200 \mu \mathrm{g} / \mathrm{ml}$ of S. quelpaertensis Nakai extracts for 24,48 and $72 \mathrm{~h}$, cells were labeled using a FITC Annexin V apoptotic assay kit (Clontech). Cells were stained for $15 \mathrm{~min}$ at room temperature in the dark with FITC-conjugated annexin V $(5 \mu \mathrm{l})$ and PI $(5 \mu \mathrm{l})$. Following incubation, the cells were analyzed in a Becton Dickinson FACScan (excitation at $488 \mathrm{~nm}$ ) equipped with CellQuest software. Early apoptotic cells were labeled with only annexin $V$, necrotic cells were stained with propidium iodide or with both annexin $\mathrm{V}$ and propidium iodide, and living cells were negative for both staining. Cells treated with argon gas served as negative controls, and those treated with $2.5 \mu \mathrm{M}$ etoposide in culture medium for $6 \mathrm{~h}$ as served as positive controls.

Cell cycle analysis. For analysis of the cell cycle profile, HCT116 cells $\left(2 \times 10^{6}\right.$ cells $/ 100-\mathrm{mm}$ dishes) were cultured for $24 \mathrm{~h}$ in McCoy's 5A medium, and the cells were harvested after 24, 48 or $72 \mathrm{~h}$ treatment with $200 \mu \mathrm{g} / \mathrm{ml}$ of $S$. quelpaertensis Nakai extracts by trypsinization and by centrifugation at $1,000 \mathrm{rpm}$ for $10 \mathrm{~min}$. The cells were washed twice with ice-cold PBS and then fixed in $70 \%(\mathrm{v} / \mathrm{v})$ ethanol overnight. Whole cells were incubated with $1 \%$ BSA (bovine serum albumin)-PBS solution containing $500 \mu \mathrm{g} / \mathrm{ml}$ PI and $10 \mu \mathrm{g} / \mathrm{ml}$ RNasse for $30 \mathrm{~min}$ at $37^{\circ} \mathrm{C}$. Cellular DNA content and apoptotic cells based on the PI signal and sub-G1 peak were measured using a Becton Dickinson FACScan (BD Bioscience). The percentage of cells in each phase of the cell cycle were determined by a Becton Dickinson FACScan (BD Bioscience) equipped with CellQuest Pro $^{\mathrm{TM}}$ software (BD Bioscience), and expressed as a percentage of cells in the respective phases.

Measurement of nitrite production. After each period of exposure, the nitrite levels in the culture media was assessed by measuring nitrite in media fractions by the Griess reaction (22). Nitrite concentrations were determined from a standard curve using sodium nitrite and the values were expressed as pmoles per $10^{9}$ viable (trypan blue-excluding) cells.

RNA isolation and semi-quantitative RT-PCR analysis. Total RNA was extracted according to the Tri Reagent (Sigma-Aldrich) supplier's protocol. The RNA was resuspended in RNase-free buffer, the concentration and purity were measured by UV spectrophotometer at 260 and $280 \mathrm{~nm}$. Total RNA was reverse transcribed using the TOP script ${ }^{\mathrm{TM}}$ one-step RT PCR kit (Enzynomics) as described previously (22). In brief, $1 \mu \mathrm{g}$ total RNA, forward and reverse oligo(dT) ${ }_{15}$ primers (20 pmol. each) (Table I), $5 \mu 1$ one-step RT PCR DyeMix, 25 units of ribonuclease inhibitor, sterile water were added to a final volume of $20 \mu \mathrm{l}$. The conditions for each PCR were $95^{\circ} \mathrm{C}$ for $30 \mathrm{sec}, 55^{\circ} \mathrm{C}$ for $30 \mathrm{sec}, 72^{\circ} \mathrm{C}$ for $1 \mathrm{~min}$ for a total of 35 cycles. In all cases RNA samples were tested 
Table I. Oligonucleotides used in semi-quantitative reverse transcription PCR.

\begin{tabular}{ll}
\hline Gene name & \multicolumn{1}{c}{ Sequence (5'-3') } \\
\hline eNOS & Sense: CCAGCTAGCCAAAGTCACCAT \\
& Antisense: GTCTCGGAGCCATACAGGATT \\
iNOS & Sense: CCAGTGACACAGGATGACCTTCAG \\
& Antisense: TGCCATTGTTGGTGG AGTAACG \\
nNOS & Sense: TTGGGGGCCTGGGATTTCTGG \\
& Antisense: GTTGGCATGGGGGAGTGAGC \\
Survivin & Sense: GCATGGGTGCCCCGACGTTG \\
& Antisense: GCTCCGGCCAGAGGCCTCAA \\
CIAP-1 & Sense: AAGTTCCTACCCCTGTCCAATG \\
& Antisense: CAAGTAGATGAGGGTAACTGGC \\
CIAP-2 & Sense: CCTGTGGTTAATCTGCCAATG \\
& Antisense: CAATTCGGCACCATAACTCTG \\
XIAP & Sense: ACACCATATACCCGAGGAAC \\
& Antisense: CTTGCATACTGTCTTCTGAGC \\
$\beta$-actin & Sense: GGTCATCTTCTCGCGGTTGGCCTT \\
& GGGGT \\
& Antisense: CCCCAGGCACCAGGGCGTGAT
\end{tabular}

NOS, endothelial NOS; iNOS, inducible NOS; nNOS, neuronal NOS; CIAP, cellular inhibitor of apoptosis; XIAP, X-linked inhibitor of apoptosis.

for their ability to generate a PCR signal by using positive control $\beta$-actin primers from Bionics. The resulting cDNA was visualized by $1.5 \%$ agarose gel electrophoresis following by ethidium bromide. The primer sequences used in semiquantitative RT-PCR analysis were listed in Table I.

Protein isolation and western blot analysis. The cells were collected after treatment and lysed with $450 \mu 1$ of iced-cold RIPA lysis buffer by incubating for $30-60 \mathrm{~min}$ at $4^{\circ} \mathrm{C}$. The lysates were centrifuged at $10,000 \times \mathrm{g}$ for $10 \mathrm{~min}$ at $4^{\circ} \mathrm{C}$, and the protein concentrations were determined using the Bio-Rad Protein Assay kit (Bio-Rad Laboratories Inc.). Equal amounts of total proteins $(50 \mu \mathrm{g})$ were mixed with loading buffer, senatured, and separated on $15 \%$ sodium dodecylsulfate polyacrylamide gel electrophoresis gels, and then blotted onto polyvinylidene difluoride (PVDF) membrane (Bio-Rad). The membranes were blocked with $5 \%$ non-fat milk at room temperature for $1 \mathrm{~h}$, and incubated with specific primary antibodies at room temperature for $2 \mathrm{~h}$. The primary antibodies used in this study were as follows: Survivin $(1: 1,000)$; CIAP-1 $(1: 1,000)$; CIAP-2 $(1: 1,000)$; XIAP $(1: 1,000)$; Bcl-2 $(1: 1,000)$; PARP $(1: 1,000)$, or $\beta$-actin $(1: 10,000)$. After washing with TBST (TBS containing $0.05 \%$ Tween-20, $\mathrm{pH} 7.6$ ) three times, the membranes were incubated with the corresponding peroxidase-conjugated secondary goat anti-rabbit or mouse IgG (diluted 1:8,000) for $1 \mathrm{~h}$ at room temperature. Following washing with TBST six times, the proteins signal was detected using Hyperfilm ECL. Densitometric analyses of resultant western blots were performed with a ChemiDoc MP Imaging System (Bio-Rad Laboratories).
Caspase 3 activity assay. The enzymatic activity of caspases induced by sodium butyrate was recorded using an ApoAlert caspase-3 colorimetric assay kit based on the manufacturer's protocol (Clontech Laboratories). Briefly, two million cells were lysed in a lysis buffer for $10 \mathrm{~min}$ on an ice bath. The lysed cells were centrifuged at $16,000 \mathrm{x} \mathrm{g}$ for $10 \mathrm{~min}$ at $4^{\circ} \mathrm{C}$, and $100 \mu \mathrm{g}$ protein was incubated with $50 \mu \mathrm{l}$ of $2 \mathrm{x}$ reaction buffer/DTT Mix and $50 \mu \mathrm{M}$ of caspase 3 substrate DEVD-pNA at $37^{\circ} \mathrm{C}$ for $1-3 \mathrm{~h}$. The optical density of the reaction mixture was measured by changes in absorbance at $405 \mathrm{~nm}$ using a $\mu$ Quant plate reader from Biotek Instruments Inc.

Statistical analysis. The data are presented as the mean \pm SD. Statistical significances were analyzed by one-way analysis of variance with post hoc Dunnett's test. $\mathrm{P}<0.05$ was considered to indicate a statistically significant difference (SPSS v.12.0) at a significance level of $\mathrm{P}<0.05$ and $\mathrm{P}<0.01$.

\section{Results}

S. quelpaertensis Nakai inhibits the proliferation of human colorectal cancer cells. We first evaluated the cytotoxic effects of $S$.quelpaertensis Nakai extracts in HCT 116 human colon carcinoma cells. Both p53-WT and p53-null cells were treated with $0,25,50,100$ and $200 \mu \mathrm{g} / \mathrm{ml}$ of $S$. quelpaertensis Nakai for 24, 48 and 72 h responded similarly, with respect to viability, in that both treatments decreased the percentage of viable cells dose- and time-dependently (Fig. 1A and B). The DMSO vehicle did not affect cell viability relative to colon cancer cells cultured in medium alone. Maximum curcumin-induced cytotoxicity was evident after $72 \mathrm{~h}$ exposure to $200 \mu \mathrm{g} / \mathrm{ml}$ of $S$. quelpaertensis Nakai extracts $(\mathrm{P}<0.01)$. Exposure to $200 \mu \mathrm{g} / \mathrm{ml}$ S. quelpaertensis Nakai for $72 \mathrm{~h}$, reduced viability in p53-WT and p53-null cells to 16 and $14 \%$, respectively, whereas comparable values after treatment with $200 \mu \mathrm{g} / \mathrm{ml} \mathrm{S}$. quelpaertensis Nakai extracts for $24 \mathrm{~h}$, were 50 and $60 \%$ (Fig. 1A and B). Fig. 1C shows that there was no significant difference between $S$. quelpaertensis Nakai extracts-treated p53-WT and p53-null cells with respect to cell viability at $200 \mu \mathrm{g} / \mathrm{ml}$, implying that $\mathrm{p} 53$ activation was not required for $S$. quelpaertensis Nakai extracts-induced cytotoxicity.

S. quelpaertensis Nakai induces the apoptosis of p53-WT and p53-null HCT116 cells. Fig. 2 shows that $200 \mu \mathrm{g} / \mathrm{ml}$ of S. quelpaertensis Nakai extracts-induced apoptosis in each of the cell types. Approximately 19.5 and $23.5 \%$ of p53-WT cells were apoptotic after $S$. quelpaertensis Nakai extracts treatment for 24 and $48 \mathrm{~h}$, respectively (8.6- and 10.4-fold, respectively, over control level) (Fig. 2). These apoptotic cell deaths by $S$. quelpaertensis Nakai extracts were observed to be approximately the same in p53-null cells as well (Fig. 2). Treatment with $200 \mu \mathrm{g} / \mathrm{ml}$ of $S$. quelpaertensis Nakai extracts for 24 and 48 h resulted in 17.7 and $26.6 \%$ of apoptosis, respectively (6.8- and 10.2-fold increases, $\mathrm{P}<0.05$ and $\mathrm{P}<0.01$; Fig. 2). A stronger apoptotic response was induced by $S$. quelpaertensis Nakai extracts treatment for $72 \mathrm{~h}$, inducing maximum frequencies of 47.3 and $52.4 \%$ in both cell lines (20.8- and 20.1-fold elevation over controls, $\mathrm{P}<0.01$; Fig. 2). 

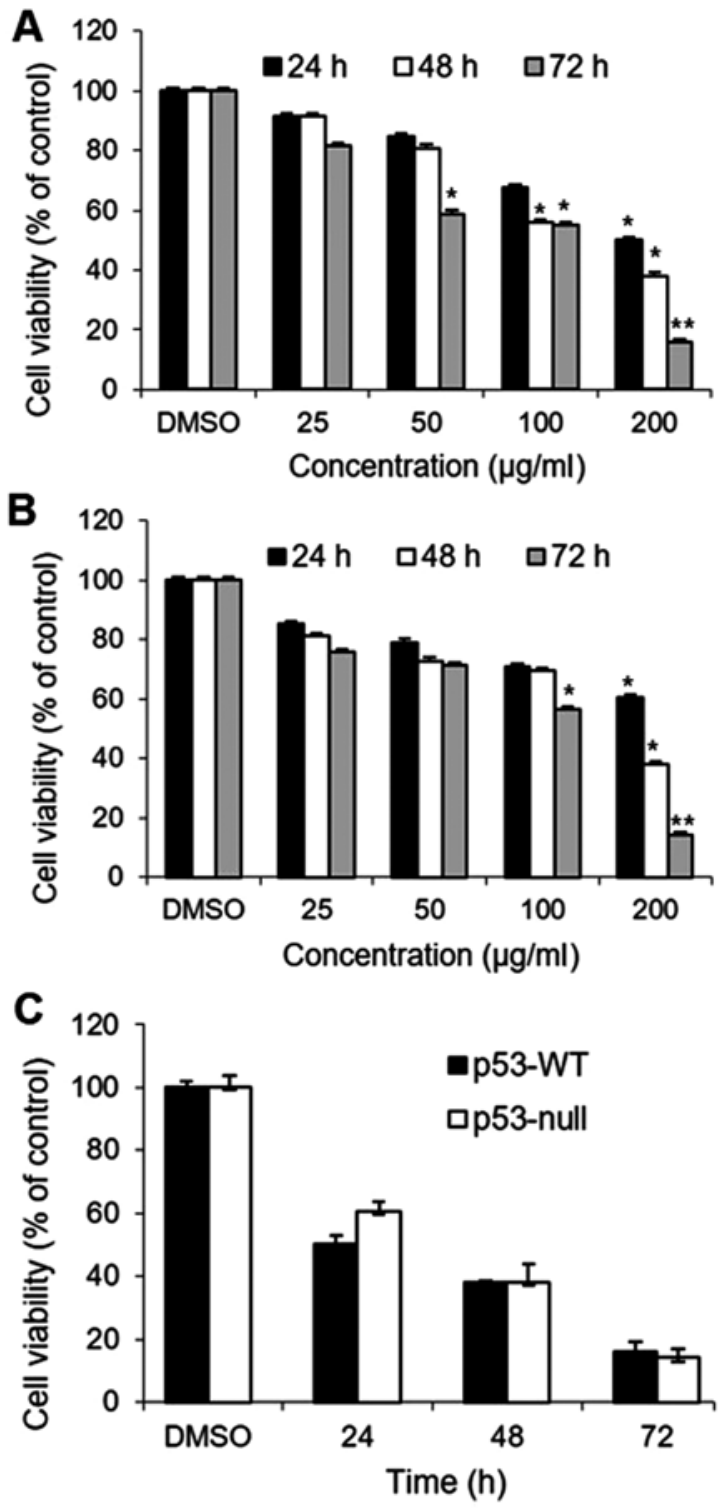

Figure 1. Percentage of viable p53-WT and p53-null HCT116 cells after treatment with $S$. quelpaertensis Nakai extracts. Cell viability was determined by trypan blue exclusion assay following treatment with $0,25,50$, 100 and $200 \mu \mathrm{g} / \mathrm{ml}$ S. quelpaertensis Nakai extracts for 24,48 and $72 \mathrm{~h}$ in (A) p53-WT and (B) p53-null HCT116 colon cancer cells. (C) S. quelpaertensis Nakai extracts is equally cytotoxic to p53-WT and p53-null cells at $200 \mu \mathrm{g} / \mathrm{ml}$ for 24,48 and $72 \mathrm{~h}$. The statistical significance of the results was analyzed by one-way ANOVA and post hoc Dunnett's test. ${ }^{*} \mathrm{P}<0.05$ and ${ }^{* *} \mathrm{P}<0.01$ vs. DMSO control. Data are presented as the mean $\pm \mathrm{SD}$ of three experiments. S. quelpaertensis, Sasa quelpaertensis; WT, wild type.

S. quelpaertensis Nakai regulates cell cycle arrest along with increase of sub-Gl population. To examine the appearance of the sub-G1 fraction, an indicator of apoptotic cell death, PI staining of DNA and flow cytometry were done in $200 \mu \mathrm{g} / \mathrm{ml}$ of S. quelpaertensis Nakai extracts-treated p53-WT and p53-null cells. S. quelpaertensis Nakai extracts significantly increase the appearance of sub-G1 fraction from 2.1 and $1.9 \%$ under control conditions to 30.5 and $31 \%$ after $72 \mathrm{~h}$ of treatment in p53-WT and p53-null cells $(\mathrm{P}<0.01)$, respectively, indicating an increase of $S$. quelpaertensis Nakai extracts-induced apoptotic cell death (Table II). Furthermore, the stage at which growth inhibition induced by with $S$. quelpaertensis Nakai extracts occurs in the p53-WT and p53-null HCT116 cell
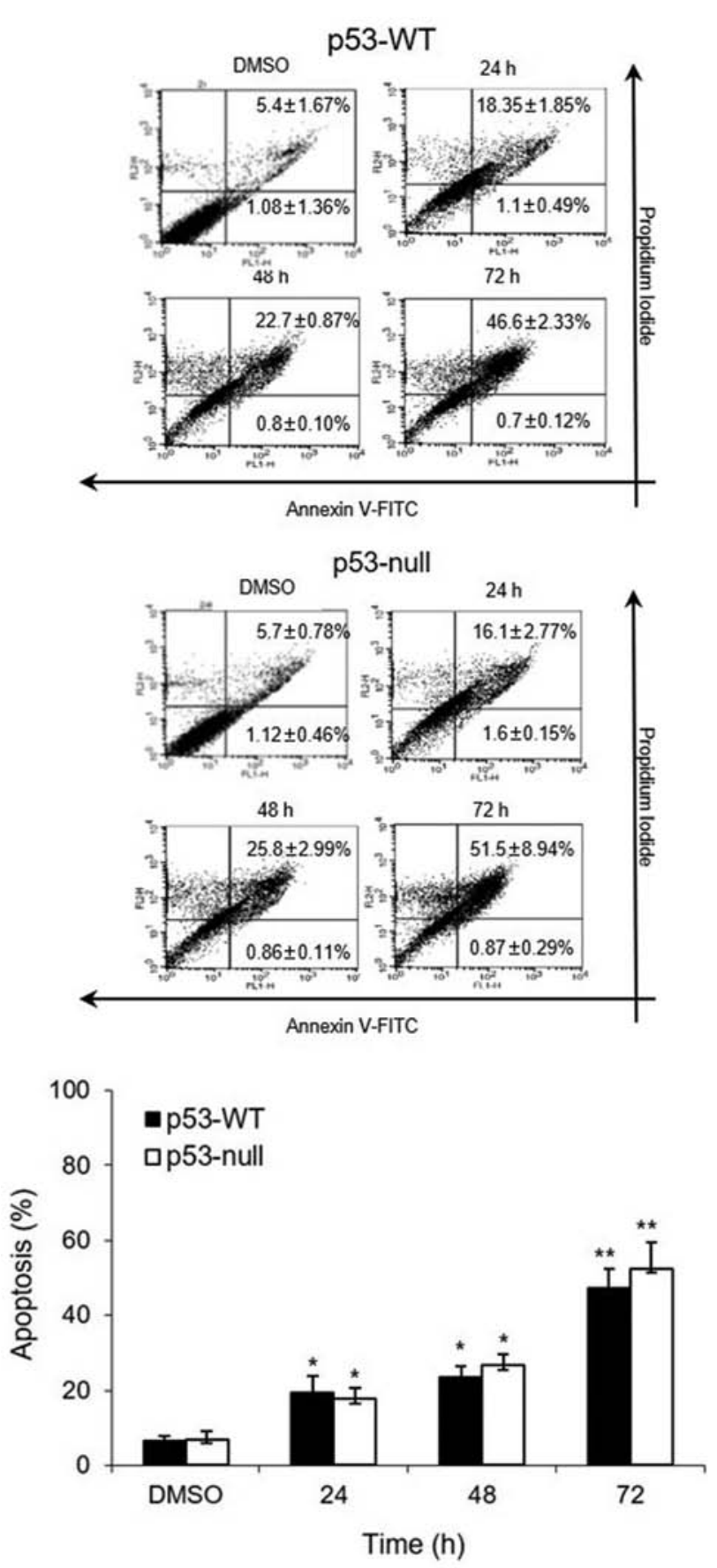

Figure 2. Induction of apoptosis by $S$. quelpaertensis Nakai extract. Apoptosis was determined by annexin $\mathrm{V}$ vs. propidium iodide staining in p53-WT and p53-null HCT116 cells treated with $200 \mu \mathrm{g} / \mathrm{ml}$ S. quelpaertensis Nakai for 24, 48 and $72 \mathrm{~h}$, respectively. Data are presented as the mean of three independent experiments \pm SD. The statistical significance of the results was analyzed by one-way ANOVA and post hoc Dunnett's test. ${ }^{*} \mathrm{P}<0.05$ and ${ }^{* *} \mathrm{P}<0.01$ vs. DMSO. S. quelpaertensis, Sasa quelpaertensis; WT, wild type.

cycle progressions were determined, with cellular distribution in the different phases the treatment (Table II). In DMSO controls, flow cytometry analysis showed 76.6 and $75.4 \%$ of cells in G0/G1 phase, 4.8 and $5.6 \%$ of cells in S phase, and 13.6 and $13.5 \%$ of cells in $\mathrm{G} 2 / \mathrm{M}$ phase in $\mathrm{p} 53$-WT and $\mathrm{p} 53$-null HCT116 cells, respectively. In contrast, in cells treated with $200 \mu \mathrm{g} / \mathrm{ml}$ of $S$. quelpaertensis Nakai extracts for $72 \mathrm{~h}$, the proportions of cells in $\mathrm{G} 0 / \mathrm{G} 1, \mathrm{~S}$, and $\mathrm{G} 2 / \mathrm{M}$ phases were 
Table II. Effect of S. quelpaertensis Nakai extracts $(200 \mu \mathrm{g} / \mathrm{ml})$ on cell cycle distribution in p53-WT and p53-null HCT116 cells.

\begin{tabular}{ccccc}
\hline & $\begin{array}{c}\text { Apoptotic } \\
\text { cells (\%) }\end{array}$ & \multicolumn{3}{c}{ Non-apoptotic cells (\%) } \\
\cline { 3 - 5 } Treatment & \begin{tabular}{c} 
(sub-G1) \\
\cline { 3 - 5 }
\end{tabular} & G0/G1 & $\mathrm{S}$ & $\mathrm{G} 2 / \mathrm{M}$ \\
\hline p53-WT & & & & \\
DMSO & $2.1 \pm 0.33$ & $76.6 \pm 6.31$ & $4.8 \pm 2.28$ & $13.6 \pm 11.24$ \\
24 h & $10.0 \pm 0.97^{\mathrm{a}}$ & $48.9 \pm 1.83^{\mathrm{a}}$ & $8.1 \pm 0.33$ & $27.0 \pm 2.25$ \\
$48 \mathrm{~h}$ & $20.2 \pm 4.31^{\mathrm{a}}$ & $37.0 \pm 2.91^{\mathrm{a}}$ & $7.7 \pm 0.37$ & $28.0 \pm 2.00^{\mathrm{a}}$ \\
$72 \mathrm{~h}$ & $30.5 \pm 4.9^{\mathrm{b}}$ & $15.7 \pm 0.38^{\mathrm{b}}$ & $14.3 \pm 0.54^{\mathrm{a}}$ & $35.7 \pm 2.26^{\mathrm{a}}$ \\
P53-null & & & & \\
DMSO & $1.9 \pm 0.39$ & $75.4 \pm 2.29$ & $5.6 \pm 0.51$ & $13.5 \pm 0.95$ \\
$24 \mathrm{~h}$ & $12.0 \pm 0.70^{\mathrm{a}}$ & $47.1 \pm 1.90^{\mathrm{a}}$ & $6.6 \pm 0.52$ & $23.9 \pm 1.97$ \\
$48 \mathrm{~h}$ & $19.2 \pm 1.25^{\mathrm{a}}$ & $34.2 \pm 2.45^{\mathrm{a}}$ & $8.4 \pm 0.20$ & $24.3 \pm 8.06^{\mathrm{a}}$ \\
$72 \mathrm{~h}$ & $31.0 \pm 2.07^{\mathrm{b}}$ & $12.0 \pm 3.00^{\mathrm{b}}$ & $16.6 \pm 0.72^{\mathrm{a}}$ & $29.8 \pm 9.75^{\mathrm{a}}$ \\
\hline
\end{tabular}

${ }^{\mathrm{a}} \mathrm{P}<0.05$ and ${ }^{\mathrm{b}} \mathrm{P}<0.01$ vs. DMSO control (one-way ANOVA and post hoc Dunnett's test). Cellular distribution (as percentage) in different phases of the cell cycle (sub-G1, G0/G1, S and G2/M) after treatment with $200 \mu \mathrm{g} / \mathrm{ml}$ S. quelpaertensis Nakai extracts is shown. Apoptotic nuclei were identified as a subploid DNA peak and distinguished from cell debris on the basis of forward light scatter and propidium iodide fluorescence. Results are presented as the mean $\pm \mathrm{SD}$ of three assays. S. quelpaertensis, Sasa quelpaertensis; WT, wild type.

15.7 and $12 \%, 14.3$ and $16.6 \%$, and 35.7 and $29.87 \%$ in p53-WT and p53-null cells, respectively. These results showed that the percentage of $\mathrm{S}$ and $\mathrm{G} 2 / \mathrm{M}$ phases cells increased, while those in the G1 phase decreased after treatment with $S$. quelpaertensis Nakai $(\mathrm{P}<0.05$ and $\mathrm{P}<0.01)$, suggesting that it promotes cell growth inhibition by inducing $\mathrm{S}$ and $\mathrm{G} 2 / \mathrm{M}$ phase arrests in both p53-WT and p53-null HCT116 cells (Table II).

Effects of S. quelpaertensis Nakai on nitrite production and NOS isoenzymes expression. The effect of $S$. quelpaertensis Nakai on three isoforms of NOS (eNOS, iNOS and nNOS) mRNA expression was evaluated by use of semiquantitative RT-PCR (Fig. 3A). RT-PCR analysis showed that after 48 and $72 \mathrm{~h}$ treatment with $200 \mu \mathrm{g} / \mathrm{ml}$ of S. quelpaertensis Nakai induced increases in each NOS isoform in p53-WT and p53-null cells, with apparently equal potency (Fig. 3A). Expression of the $\beta$-actin gene and production of its mRNA was not altered during any of the treatments (Fig. 3A). These findings are consistent with the time-dependent increases in nitrite production were observed in both cell type in response to S. quelpaertensis Nakai extracts treatments (Fig. 3B).

S. quelpaertensis Nakai regulated expression of inhibitors of apoptosis (IAP) family. In this study, we subsequently investigated mechanism underlying cell death induced by $S$. quelpaertensis Nakai by RT-PCR and western blotting, with a focus upon antiapoptotic activity of IAP family member (Fig. 4). Compared with controls, the CIAP-1 and CIAP-2 mRNA expression were remarkably down-regulated after treated with $S$. quelpaertensis Nakai extracts for

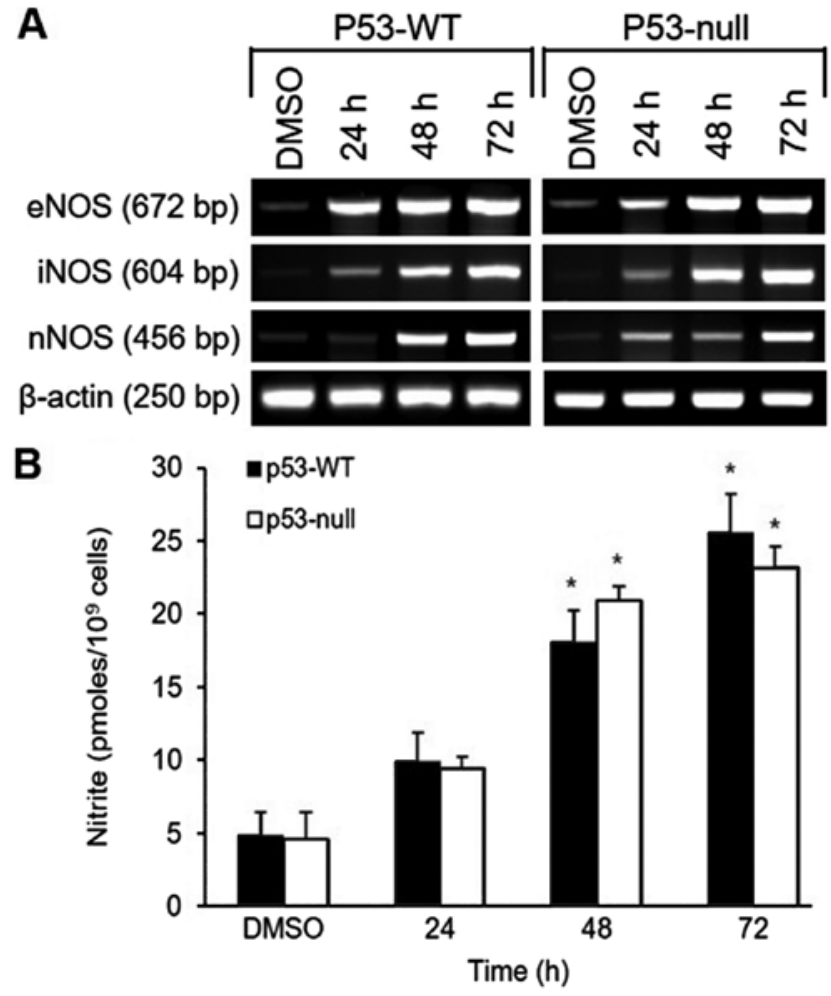

Figure 3. Effect of NO on S.quelpaertensis Nakai-induced apoptosis in human colon cancer cells. Effects of S. quelpaertensis Nakai extract on (A) NOS activity and (B) cellular nitrite level in p53-WT and p53-null HCT116 cells treated with $200 \mu \mathrm{g} / \mathrm{ml}$ S. quelpaertensis Nakai for 24, 48 and $72 \mathrm{~h}$. Data shown are representative of three independent experiments. The statistical significance of the results was analyzed by one-way ANOVA and post hoc Dunnett's test. " $\mathrm{P}<0.05$ vs. DMSO. S. quelpaertensis, Sasa quelpaertensis; WT, wild type; eNOS, endothelial NOS; iNOS, inducible NOS; nNOS, neuronal NOS.

24, 48 and $72 \mathrm{~h}$, while the level of Survivin and XIAP mRNA expression did not decrease until both p53-WT and p53-null cells were exposed to $S$. quelpaertensis Nakai extracts for $72 \mathrm{~h}$ (Fig. 4A). Moreover, treatment of the cells with S. quelpaertensis Nakai extracts for 24, 48 and $72 \mathrm{~h}$ led to a reduction of Survivin, CIAP-1 and CIAP-2 proteins in both p53-WT and p53-null HCT116 cells (Fig. 4B). In contrast, no apparent changes of XIAP mRNA and protein expressions were found in S. quelpaertensis Nakai-treated group compared with DMSO controls (Fig. 4A and B).

Effects of S. quelpaertensis Nakai treatment on Bcl-2. PARP expression and caspase 3 activity. The levels of Bcl-2 and PARP protein expression in HCT116 cells were analyzed by western blotting. The p53-WT and p53-null cells were treated with $200 \mu \mathrm{g} / \mathrm{ml}$ of S. quelpaertensis Nakai extracts for 24, 48 and $72 \mathrm{~h}$. Compared with the control group, treatment with $S$. quelpaertensis Nakai downregulated the expression of Bcl-2, whereas the levels of PARP were upregulated in both cells (Fig. 5A). To further determine the apoptotic cell death induced by $S$. quelpaertensis Nakai, HCT116 cells were left untreated or treated with $200 \mu \mathrm{g} / \mathrm{ml}$ of $S$. quelpaertensis Nakai extracts for 24, 48 and $72 \mathrm{~h}$, and caspase- 3 activity assay in a colorimetric assay based on the cleavage of the synthetic peptide Ac-DEVD-pNA was done (Fig. 5B). S. quelpaertensis Nakai increased caspase- 3 activity by approximately 2 -fold (Fig. 5B). 


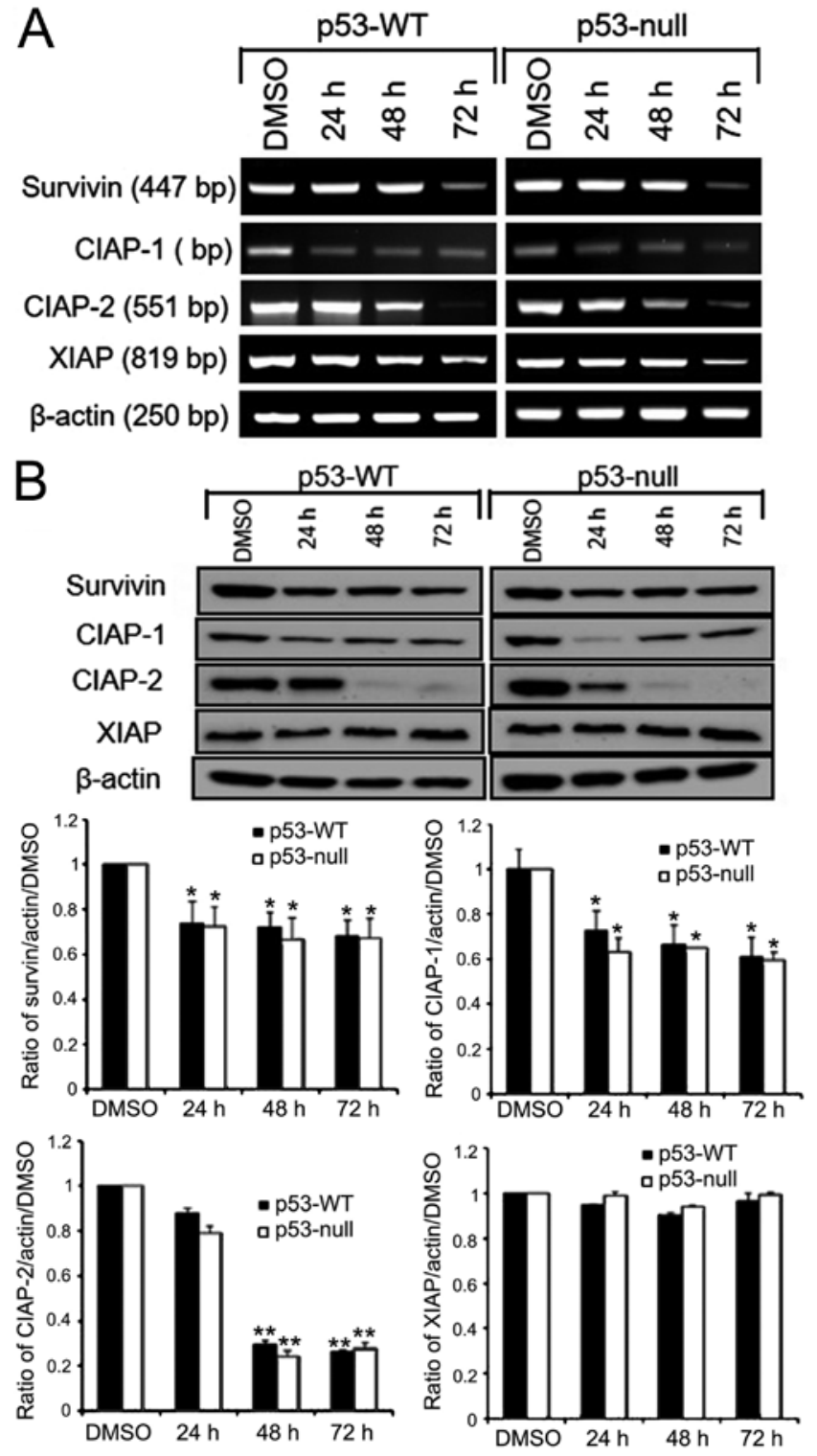

Figure 4. mRNA and protein expression levels of IAP in human colon cancer cells after treatment with $S$. quelpaertensis Nakai. (A) Reverse transcription PCR and (B) western blot analyses of the IAP family (Survivin, CIAP-1, CIAP-2 and XIAP) mRNA and protein levels in p53-WT and p53-null HCT116 cells treated with $200 \mu \mathrm{g} / \mathrm{ml} \mathrm{S}$. quelpaertensis Nakai extract for 24,48 and $72 \mathrm{~h}$. Semi-quantitative PCR was performed using primers specific to survivin, CIAP-1, CIAP-2 and XIAP or a $\beta$-actin control on $1 \mu \mathrm{g}$ total RNA prepared. Additionally, cell lysates were prepared and subjected to western blot analysis using specific antibodies. Band intensities were calculated by densitometric analysis and normalized to actin levels. Typical results from three independent experiments are shown. The statistical significance of the results was analyzed by one-way ANOVA and post hoc Dunnett's test. "P<0.05 and ${ }^{* *} \mathrm{P}<0.01$ vs. DMSO. S. quelpaertensis, Sasa quelpaertensis; WT, wild type; CIAP, cellular inhibitor of apoptosis; XIAP, X-linked inhibitor of apoptosis.

\section{Discussion}

Cancer is affecting millions of people every year and our emphasis is to explore appropriate natural sources and to suggest a novel anticancer candidate that can combat cancer in a better way. We have recently reported antioxidant and anticancer activities of the ethanol and water extracts of Sasa quelpaertensis Nakai leaves $(19,23)$. The results showed that both extracts showed antioxidant activities with different
A
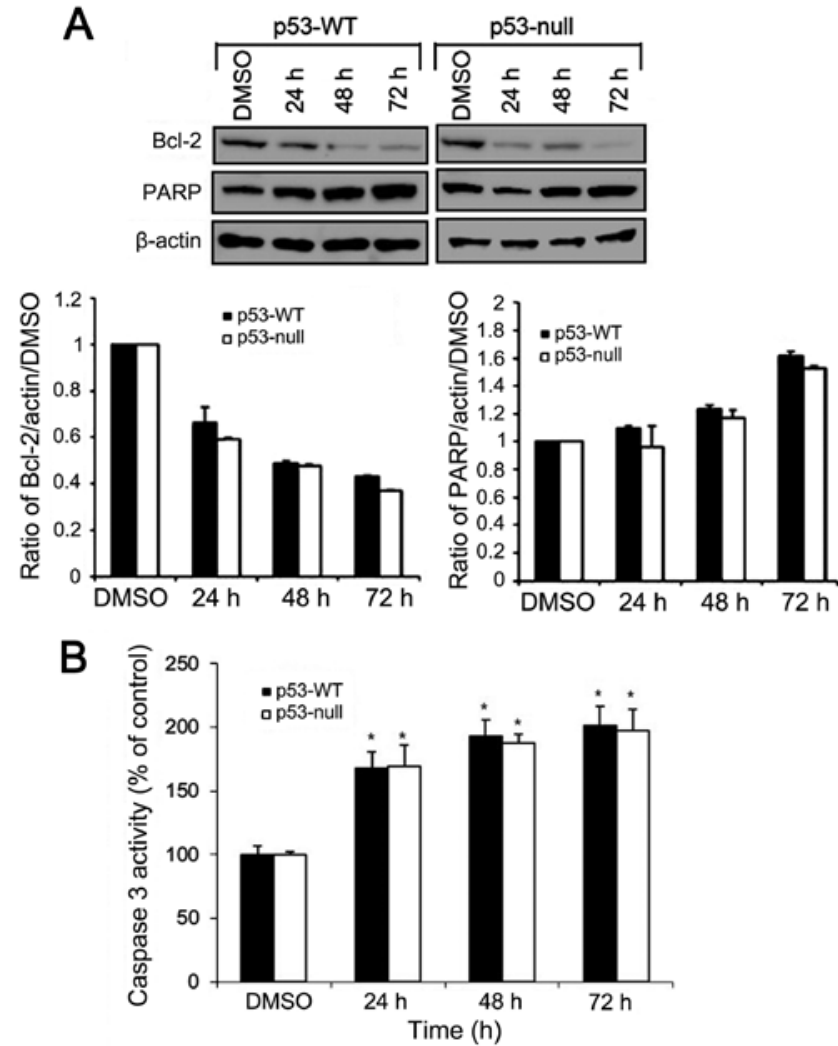

Figure 5. Protein expression levels of Bcl-2 and PARP, and caspase 3 activity in human colon cancer cells after treatment with $S$. quelpaertensis Nakai. Representative western blot images showing alterations in the levels of (A) Bcl-2 and PARP and (B) caspase 3 activity in p53-WT and p53-null HCT116 cells treated with $200 \mu \mathrm{g} / \mathrm{ml} \mathrm{S}$. quelpaertensis Nakai extract for 24, 48 and $72 \mathrm{~h}$. Band intensities were calculated by densitometric analysis and normalized to actin levels. Data are presented as the mean of three independent experiments \pm SD. The statistical significance of the results was analyzed by one-way ANOVA and post hoc Dunnett's test. ${ }^{*} \mathrm{P}<0.05$ vs. DMSO. PARP, poly(ADP-ribose) polymerase 1; S. quelpaertensis, Sasa quelpaertensis; WT, wild type.

magnitudes of potency. The ethanol extracts of S. quelpaertensis Nakai contained a larger quantity of phenolics and flavonoids (2.1- and 4.6-fold, respectively) and exhibited stronger radical scavenging, ferrous chelating and reducing power abilities, and an anti-proliferative effect on HCT116 colon cancer cells when compared to water extracts (23). In addition, other studies revealed that $S$. quelpaertensis Nakai extracts showed antiproliferation and apoptosis induction properties in human leukemia HL-60 cells (17), as well as in human and lung cancer A549 and H1299 cells. Despite previous reports demonstrated that potential clinical application of dwarf bamboo S. quelpaertensis Nakai extracts for prevention or treatment of neoplastic disease $(19,23,24)$, the underlying mechanism has not been clarified.

The aim of the present study was to investigate molecular mechanisms of cell death induced by $S$. quelpaertensis Nakai extracts in HCT116 colon carcinoma cells. In the present study, we observed that $S$.quelpaertensis Nakai decrease of cell viability with increasing concentrations at different treatment times in p53-WT and p53-null HCT116 cells. Flow cytometric analysis suggested that apoptosis was a major contributor to cell death induced by $S$. quelpaertensis Nakai in both cell types; nearly $47 \%$ of p53-WT and 52\% of p53-null cells stained with 
$\mathrm{PI}$ and annexin V $72 \mathrm{~h}$ after $S$. quelpaertensis Nakai treatment $(\mathrm{P}<0.01)$. DNA content measurement by Cell cycle analysis showed a remarkable accumulation of subploid cells in the sub-G1 area in both p53-WT and p53-null cells after treatment with $S$.quelpaertensis Nakai for $72 \mathrm{~h}$ when compared with the DMSO control group $(\mathrm{P}<0.01$; Table II). Since accumulation of the sub-G1 peaks indicated characteristics of apoptosis, our results provide strong evidence for cytotoxicity induced by with $S$. quelpaertensis Nakai resulting in the decrease of the number of viable cells.

Nitric oxide (NO') is a free radical messenger molecule that plays a crucial role in controlling various physiological functions in vivo $(25,26)$. This molecule is produced by three different isoforms of the enzyme nitric oxide synthase (NOS) which can regulate biological activity in a variety of cells (25). $\mathrm{NO}^{\bullet}$ has also been shown to be involved in many of the pathophysiological processes that contribute to the development and progression of cancer (27). Based on the existing literature, it is clear that $\mathrm{NO}^{-}$may be viewed as a double-edged sword in cancer $(27,28)$. High concentrations of $\mathrm{NO}^{*}$ may mediate cancer cell apoptosis and the inhibition of cancer growth, whereas cancer growth and proliferation is promoted at low concentrations of $\mathrm{NO}^{*}$. The regulation of cancer growth by $\mathrm{NO}^{*}$ represents an important player in cancer research, including colon cancer $(25,27,28)$. In this study, S. quelpaertensis Nakai extracts caused upregulation of all endogenous NOS activities and, in turn, an increase in $\mathrm{NO}^{*}$ production leading to cell death. Seventy two h after treatment with $S$. quelpaertensis Nakai induced the highest level of NOS expression and 4.7to 5.5-fold higher $\mathrm{NO}^{\circ}$ production, compared with compared with DMSO control, indicating a direct relationship between increased $\mathrm{NO}^{\circ}$ production and the loss of cell viability, caused by $S$. quelpaertensis Nakai.

Because mechanisms through which S. quelpaertensis Nakai extracts induce cell death are poorly understood, we here investigated their effects on apoptotic signaling pathways. In the current study, treatment of the cells with $200 \mu \mathrm{g} / \mathrm{ml}$ of $S$. quelpaertensis Nakai for 24, 48 and $72 \mathrm{~h}$ led to a reduction of survivin, CIAP-1, CIAP-2 and XIAP in both p53-WT and p53-null HCT116 cells, suggesting the high expression of these IAPs in human colon cancer cells may act as a contributing factor to resistance by $S$. quelpaertensis Nakai. Activation of the nuclear factor $\kappa \mathrm{B}(\mathrm{NF}-\kappa \mathrm{B})$ transcription factor plays an important role in inhibition of apoptotic pathway (29). Apoptosis-regulatory IAP family such as survivin, CIAP-1, CIAP-2 and XIAP is transcriptionally regulated by $\mathrm{NF}-\kappa \mathrm{B}(30)$. These IAPs have been reported to block apoptosis by direct binding to caspases such as caspase-3 and caspase-9, indicating that expression of IAPs under the control of $\mathrm{NF}-\kappa \mathrm{B}$ plays an important role in the anti-apoptotic pathway (30).

Here we also observed an increase in the PARP and caspase 3 activity in $S$. quelpaertensis Nakai extracts-treated colon cancer cells while suppressing expression of anti-apoptotic proteins such as Bcl-2, implying apoptosis induction that involved downregulation of Bcl-2 and cleavage of PARP, and its mechanism may be associated with the Bcl-2/caspase-3 signaling pathway. Caspase-3 is a family of cysteine proteases and plays a crucial role in the execution phase of apoptosis, and that its activation often marks the commitment to apoptosis $(31,32)$. Bcl-2 inhibits cytochrome c release from mitochondria as well as caspase-3 $(32,33)$. PARP is cleaved by caspase- 3 , which causes apoptosis $(32,34)$. Our findings are therefore consistent with other reports that $S$. quelpaertensis Nakai triggers apoptosis in human leukemia HL-60 $(16,17)$ and gastric cancer MKN-74 (17) cells by up-regulation of Bax, caspase 3 and PARP as well as down-regulation of anti-apoptotic proteins such as survivin and Bcl-2.

The p53 tumor suppressor gene is a critical regulator of cell survival and proliferation, activated by cellular stresses including DNA damage, oncogene activation and cytotoxic agents (35-37). The loss of p53 activity promotes tumorigenesis in various organs including colon (38). Generally, tumor therapy including radiotherapy and chemotherapy, which can induce p53-mediated promotion in tumor cells (35-37). Although disruption of p53 expression generally facilitates cancer cell resistance to chemotherapy in some studies, it might not be concluded that p53 negative tumors are always less sensitive to these drugs $(38,39)$. The effects of p53 on chemosensitivity can be dependent both on external stimulation types and on internal genetic environment of the cells (38). In the present study, we systematically explored the possible role of p53 in the proapoptotic activity of $S$. quelpaertensis Nakai using p53-isogenic pair of colon cancer cell-lines and demonstrated that exposure to $S$. quelpaertensis Nakai caused apoptosis in colon cancer cells indiscriminately of p53 status since a similar cytotoxic and apoptotic effects were observed in p53-WT and p53-null HCT116 cells, demonstrating that p53 is not the only determinant of the fate of $S$. quelpaertensis Nakai-treated colon cancer cells. This might be possibly explained by the induction of apoptosis proceeds through a caspase-mediated mitochondria amplification regardless of p53 status.

Taken together, our results indicate that $S$. quelpaertensis Nakai-induced apoptosis in HCT 116 colon cancer cells was independent of p53 expression. Furthermore, this study demonstrates that oxidative stress as a result of $\mathrm{NO}^{\circ}$ production triggered p53-independent apoptosis. Therapeutic application of $S$. quelpaertensis Nakai is therefore predicted to be effective against colon cancers cells irrespective of their p53 status.

\section{Acknowledgements}

The author would like to thank Professor Gerald Norman Wogan (Department of Biological Engineering, Massachusetts Institute of Technology) for providing human colon cancer cell lines.

\section{Funding}

The present study was supported by the Basic Science Research Program (grant nos. 2017R1D1A1B03028849, 2016R1A6A1A03012862 and 2014R1A1A2056292) through the National Research Foundation of Korea (NRF) funded by the Ministry of Education, Science and Technology, Republic of Korea.

\section{Availability of data and materials}

The datasets used and/or analyzed during the present study are available from the corresponding author on reasonable request. 


\section{Authors' contributions}

MYK conceived the study, designed the experiments, performed the experiments, analyzed the data, and wrote and approved the manuscript.

\section{Ethics approval and consent to participate}

Not applicable.

\section{Patient consent for publication}

Not applicable.

\section{Competing interests}

The author declares that they have no competing interests.

\section{References}

1. Bray F, Ferlay J, Soerjomataram I, Siegel RL, Torre LA and Jemal A: Global cancer statistics 2018: GLOBOCAN estimates of incidence and mortality worldwide for 36 cancers in 185 countries. CA Cancer J Clin 68: 394-424, 2018.

2. Palma S, Zwenger AO, Croce MV, Abba MC and Lacunza E: From molecular biology to clinical trials: Toward personalized colorectal cancer therapy. Clin Colorectal Cancer 15: 104-115, 2016.

3. Janne PA and Mayer RJ: Chemoprevention of colorectal cancer. N Engl J Med 342: 1960-1968, 2000.

4. de Gramont A, Figer A, Seymour M, Homerin M, Hmissi A, Cassidy J, Boni C, Cortes-Funes H, Cervantes A, Freyer G, et al: Leucovorin and fluorouracil with or without oxaliplatin as first-line treatment in advanced colorectal cancer. J Clin Oncol 18: 2938-2947, 2000.

5. Rejhová A, Opattová A, Čumová A, Slíva D and Vodička P: Natural compounds and combination therapy in colorectal cancer treatment. Eur J Med Chem 144: 582-594, 2018.

6. Lichota A and Gwozdzinski K: Anticancer activity of natural compounds from plant and marine environment. Int J Mol Sci 19: pii: E3533, 2018.

7. Hu C, Zhang Y and Kitts DD: Evaluation of antioxidant and prooxidant activities of bamboo Phyllostachys nigra var. Henonis leaf extract in vitro. J Agric Food Chem 48: 3170-3176, 2000

8. Kweon MH, Hwang HJ and Sung HC: Identification and antioxidant activity of novel chlorogenic acid derivatives from bamboo (Phyllostachys edulis). J Agric Food Chem 49: 4646-4655, 2001

9. Ra JH, Nakamura M, Herath KHINM, Jee Y and Kim JS Antioxidant and hepatoprotective effects of different ethanol concentrations in extraction from leaves of Sasa quelpaertensis Nakai. South African J Botany 112: 376-382, 2017.

10. Seki T and Maeda H: Cancer preventive effect of Kumaizasa bamboo leaf extracts administered prior to carcinogenesis or cancer inoculation. Anticancer Res 30: 111-118, 2010.

11. Sakai S, Saito G, Sugayama J, Kamasuka T, Takano T and Takada S: Anticancer effect of polysaccharide fraction prepared from bamboo grass. Gan 55: 197-203, 1964.

12. Yoon SA, Kang SI, Shin SH, Ko HC and Kim SJ: Anti-diabetic potential of a Sasa quelpaertensis Nakai extract in L6 skeletal muscle cells. Food Sci Biotechnol 23: 1335-1339, 2014.

13. An SM, Lee SI, Choi SW, Moon SW and Boo YC: p-Coumaric acid, a constituent of Sasa quelpaertensis Nakai, inhibits cellular melanogenesis stimulated by alpha-melanocyte stimulating hormone. Br J Dermatol 159: 292-299, 2008.

14. Madushani Herath KHIN, Bing SJ, Cho J, Kim A, Kim G, Kim JS, Kim JB, Doh YH and Jee Y: Sasa quelpaertensis leaves ameliorate alcohol-induced liver injury by attenuating oxidative stress in HepG2 cells and mice. Acta Histochem 120: 477-489, 2018.
15. Ryou SH, Kang MS, Kim KI, Kang YH and Kang JS: Effects of green tea or Sasa quelpaertensis bamboo leaves on plasma and liver lipids, erythrocyte $\mathrm{Na}$ efflux, and platelet aggregation in ovariectomized rats. Nutr Res Pract 6: 106-112, 2012.

16. Jang MG, Park SY, Lee SR, Choi SY, Hwang JH, Ko HC, Park JG, Chung WS and Kim SJ: Sasa quelpaertensis leaf extracts induce apoptosis in human leukemia HL-60 cells. Food Sci Biotechnol 17: 188-190, 2008.

17. Jang MG, Ko HC and Kim SJ: Effect of Sasa quelpaertensis Nakai extracts and its constituent p-coumaric acid on the apoptosis of human cancer cell lines. J Food Biochem 24: 293-297, 2018.

18. Byun JH and Kim MY: Apoptotic effect of Sasa quelpaertensis Nakai in human colon cancer HT-29 cells. J Life Sci 24: 1012-1018, 2014.

19. Kim JH and Kim MY: Antiproliferative and apoptotic effects of Sasa quelpaertensis Nakai in human cancer cells. J Life Sci 24: 903-909, 2014.

20. Redza-Dutordoir M and Averill-Bates DA: Activation of apoptosis signalling pathways by reactive oxygen species. Biochim Biophys Acta 1863: 2977-2992, 2016.

21. Tang D, Kang R, Berghe TV, Vandenabeele P and Kroemer G: The molecular machinery of regulated cell death. Cell Res 29: 347-364, 2019.

22. Moon SH, Cho MH and Kim MY: Cellular inactivation of nitric oxide induces p53-dependent apoptosis in human melanoma cells. Trop J Pharm Res 15: 1595-1603, 2016.

23. Kim JY, Kim JH, Byun JH, Lee YJ, Im SJ, Lee D, Moon SH and Kim MY: Antioxidant and anticancer activities of water and ethanol extracts obtained from Sasa quelpaertensis Nakai. Life Sci J 10: 1250-1254, 2013.

24. Kim M, Kim YS, Kim KM, Ko HC, Kim SJ, Kim JH and Kim Y: Combination of Sasa quelpaertensis Nakai leaf extract and cisplatin suppresses the cancer stemness and invasion of human lung cancer cells. Integr Cancer Ther 13: 529-540, 2014.

25. Xu W, Liu LZ, Loizidou M, Ahmed M and Charles IG: The role of nitric oxide in cancer. Cell Res 12: 311-320, 2002.

26. Wink DA, Ridnour LA, Hussain SP and Harris CC: The reemergence of nitric oxide and cancer. Nitric Oxide 19: 65-67, 2008.

27. Rao CV: Nitric oxide signaling in colon cancer chemoprevention. Mutat Res 555: 107-119, 2004.

28. Li CQ and Wogan GN: Nitric oxide as a modulator of apoptosis. Cancer Lett 226: 1-15, 2005.

29. Karin M and Lin A: NF-kappaB at the crossroads of life and death. Nat Immunol 3: 221-227, 2002.

30. Salvesen GS and Duckett CS: IAP proteins: Blocking the road to death's door. Nat Rev Mol Cell Biol 3: 401-410, 2002.

31. Fiandalo MV and Kyprianou N: Caspase control: Protagonists of cancer cell apoptosis. Exp Oncol 34: 165-175, 2012.

32. Jin SJ, Yang Y, Ma L, Ma BH, Ren LP, Guo LC, Wang WB, Zhang YX, Zhao ZJ and Cui M: In vivo and in vitro induction of the apoptotic effects of oxysophoridine on colorectal cancer cells via the $\mathrm{Bcl}-2 / \mathrm{Bax} /$ caspase-3 signaling pathway. Oncol Lett 14 : 8000-8006, 2017.

33. Zhao Y, Jing Z, Lv J, Zhang Z, Lin J, Cao X, Zhao Z, Liu P and Mao W: Berberine activates caspase-9/cytochrome c-mediated apoptosis to suppress triple-negative breast cancer cells in vitro and in vivo. Biomed Pharmacother 95: 18-24, 2017.

34. Koh DW, Dawson TM and Dawson VL: Mediation of cell death by poly(ADP-ribose) polymerase-1. Pharmacol Res 52: 5-14, 2005.

35. Ozaki T and Nakagawara A: p53: The attractive tumor suppressor in the cancer research field. J Biomed Biotechnol 2011: 603925, 2011.

36. Levine AJ, Momand J and Finlay CA: The p 53 tumour suppressor gene. Nature 351: 453-456, 1991.

37. Zilfou JT and Lowe SW: Tumor suppressive functions of p53. Cold Spring Harb Perspect Biol 1: a001883, 2009.

38. Chen L, Jiang J, Cheng C, Yang A, He Q, Li D and Wang Z: P53 dependent and independent apoptosis induced by lidamycin in human colorectal cancer cells. Cancer Biol Ther 6: 965-973, 2007.

39. Mahyar-Roemer M and Roemer K: p21 Waf1/Cip1 can protect human colon carcinoma cells against p53-dependent and p53-independent apoptosis induced by natural chemopreventive and therapeutic agents. Oncogene 20: 3387-3398, 2001. 